\title{
Recomendaciones clínicas del Grupo Español de Tratamiento Conservador del Cáncer de Cabeza y Cuello (GETCOCACU) para la prevención y la terapéutica de la xerostomía radioinducida
}

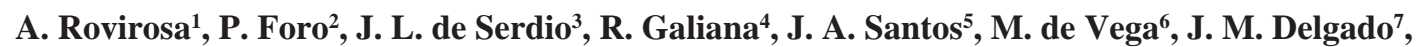 \\ A. de la Torre ${ }^{8}$, A. Ramos ${ }^{9}$, A. Villar ${ }^{10}$. \\ Comité Científico del Grupo Español de Tratamiento Conservador \\ del Cáncer de Cabeza y Cuello (GETCOCACU)
}

\section{Resumen}

La xerostomía radioinducida es un síntoma que manifiestan prácticamente la totalidad de los pacientes irradiados por cáncer de cabeza y cuello, en los que las glándulas salivares se encuentran incluidas dentro de los campos de tratamiento. Condiciona manifestaciones clínicas subjetivas y objetivas que pueden llegar a alterar de modo importante la calidad de vida de estos pacientes. Los únicos tratamientos que se han podido ofrecer hasta hace poco han sido sintomáticos (medidas de carácter general, sustitutivos de la saliva y estimulantes salivares) y se ha atribuido una eficacia no completa a los fármacos radioprotectores. Las técnicas de radioterapia conformada y de intensidad modulada, al permitir proteger a las glándulas salivares de dosis altas de radiación, son la mejor opción terapéutica en los casos que sean tributarios. La prevalencia de este síntoma y sus manifestaciones clínicas ha llevado al GETCOCACU a establecer unas recomendaciones en el manejo de la xerostomía.

\section{Palabras clave:}

Xerostomía radioinducida. Tratamiento. Prevención.

Oncología, 2005; 28 (6):267-274

\footnotetext{
${ }^{1}$ Servicio de Oncología Radioterápica. ICMHO. Hospital Clínic i Universitari. IDIBAPS. Barcelona.

2 Servicio de Oncología Radioterápica. Hospital de la Esperanza. Barcelona.

3 Servicio de Otorrinolaringología. Hospital Na Señora de la Candelaria. Santa Cruz de Tenerife.

${ }^{4}$ Servicio de Oncología Radioterápica. Institut Català d’Oncología. Hospitalet de Llobregat.

${ }^{5}$ Servicio de Oncología Radioterápica. Hospital General Universitario Gregorio Marañón. Madrid.

${ }^{6}$ Servicio de Oncología Radioterápica. Hospital de la Santa Creu i Sant Pau. Barcelona.

7 Servicio de Oncología Radioterápica. Hospital Gómez Ulla. Madrid.

${ }^{8}$ Servicio de Oncología Radioterápica. Clínica Puerta de Hierro. Madrid.

9 Servicio de Oncología Radioterápica. Hospital Ramón y Cajal. Madrid.

10 Servicio de Oncología Radioterápica. Presidente del GETCOCACU. Hospital N ${ }^{a}$ Señora de la Candelaria. Santa Cruz de Tenerife.
} 


\section{Summary}

Radiation-related xerostomia is a symptom presenting almost all patients irradiated for head and neck cancer when salivary glands are involved in the radiation field. Xerostomia causes subjective and objective manifestations strongly affecting the quality of life of the patients. Until now, the treatment has been the palliation of symptoms and the use of general measures, saliva substitutes, saliva stimulants, and littlebeneficial radioprotective drugs. Conformal radiotherapy and intensity modulated techniques permit in some cases the avoidance of salivary glands irradiation with high radiotherapy doses, so turning into the best options for these patients. The prevalence of xerostomia and the inconvenient manifestations have lead the GETCOCACU to establish recommendations for the xerostomia management and treatment.

Key words: Xerostomia after radiotherapy. Treatment. Prevention.

La xerostomía radioinducida es un síntoma que refleja la disminución del flujo salivar secundaria a la irradiación de las glándulas salivares y afecta, en mayor o menor grado, a prácticamente la totalidad de los pacientes irradiados por cáncer de cabeza y cuello. Una vez instaurada es irreversible y se asocia con el tiempo a toda una serie de problemas clínicos y síntomas de sequedad oral; el paciente tiene que aprender a vivir con las manifestaciones y consecuencias de la xerostomía ya que, hasta la actualidad, no se han descrito otros tratamientos efectivos diferentes a los paliativos. La importancia de este síntoma, poco valorado hasta hace unos años debido a la falta de un tratamiento curativo, ha llevado al Comité Científico del GETCOCACU a establecer unas recomendaciones para la práctica clínica diaria. El objetivo del presente texto es, después de realizar una introducción de las medidas terapéuticas generales paliativas y un análisis del estado actual de los tratamientos existentes, llegar a unas conclusiones en forma de recomendaciones para el manejo de la xerostomía.

La xerostomía en los pacientes irradiados por cáncer de cabeza y cuello es debida a la falta de saliva por destrucción de las glándulas salivares. Se manifiesta clínicamente cuando el flujo salivar se reduce al 50\%. Dosis de irradiación superiores a los 26 Gy ya condicionan xerostomía, que se agrava y se hace irreversible a partir de los $50 \mathrm{~Gy}$. Al finalizar la radioterapia, utilizando campos de tratamiento convencionales, un 65-89\% de los pacientes presentarán xerostomía grado 2 o superior (según la escala RTOG) y el flujo salivar se reduce, habitualmente, al $30-40 \%$ del previo al tratamiento. Se ha referido una mejoría subjetiva de la xerostomía a partir del año de la irradiación sin que ello tenga una gran repercusión en el flujo salivar, el cual se mantiene siempre en valores similares ${ }^{1-8}$.

La disminución de saliva condiciona molestias en la cavidad oral de tipo urente, disconfort al hablar, cierto dolor con la deglución alimentaria (por la escasa lubricación del bolo alimentario) y un aumento de la placa bacteriana adherida a los dientes por la falta de autolimpieza. Debido a que la saliva permite mantener un $\mathrm{pH}$ neutro en la cavidad oral y posee propiedades bactericidas ${ }^{9-15}$, cuando aparece una disminución lo suficientemente importante del flujo salivar se produce una acidificación del $\mathrm{pH}$ oral, lo que condiciona alteraciones en la flora habitual y un aumento de los gérmenes patógenos. Entre estos últimos destacan las cándidas, condicionantes de la candidiasis oral clínica durante y después de la radioterapia, y el estreptococo mu$\operatorname{tans}^{12-15}$. Un flujo salivar inferior a $0,1 \mathrm{ml} / \mathrm{min}$ ya puede causar un aumento en la incidencia de cándidas ${ }^{16}$. Asimismo, el descenso del $\mathrm{pH}$ oral produce una alteración en el equilibrio hidromineral de los dientes que, conjuntamente con la presencia del estreptococo mutans, da lugar a una mayor incidencia de caries. Estas caries son típicamente cervicales y provocan la ruptura de los dientes a ese nivel $^{17}$. 
La gravedad de la xerostomía y de sus manifestaciones clínicas variará en función del volumen irradiado de glándulas salivares mayores, de la dosis de radioterapia que reciban y también, probablemente, del número de glándulas salivares menores incluidas en los campos de tratamiento. En la actualidad todavía no está establecido y extendido el estudio de la correlación entre la dosis de radioterapia, el volumen o porcentaje irradiado de glándulas salivares y la xerostomía, con sus repercusiones clínicas. Algunos estudios efectuados, si bien con un número reducido de pacientes irradiados mediante técnicas conformacionales o de intensidad modulada, indican que el flujo salivar es recuperable cuando la dosis de radioterapia en las glándulas salivares mayores es inferior a los $26 \mathrm{~Gy}^{1-7}$.

En el análisis de la xerostomía radioinducida también debe tenerse en cuenta la posibilidad de otras posibles etiologías. Por encima de los 55 años, una cuarta parte de la población normal presenta xerostomía y sólo el $40 \%$ de ellos tiene un flujo salivar normal. La causa más importante de xerostomía a partir de los 65 años es la farmacológica, provocándola fármacos de uso tan frecuente como los antidepresivos, los hipoglucemiantes, los antihipertensivos y los hipolipemiantes, entre otros ${ }^{9,10,18}$.

\section{Recomendaciones generales para paliar la xerostomía y sus consecuencias}

Hasta hace poco tiempo, el manejo de la xerostomía radioinducida ha consistido básicamente en evitar factores que pudieran provocar o agravar la sensación de boca seca, en la administración de los sustitutivos salivares y en la prevención de las complicaciones orales asociadas $9,10,12,13,19$. Entre las medidas paliativas de carácter general destacan las siguientes.

1. Siempre que sea posible se eliminarán los medicamentos causantes de xerostomía.

2. Antes de iniciar la radioterapia, los pacientes deberán visitar a su estomatólogo/odontólogo a fin de eliminar las piezas dentarias en mal estado, practicar endodoncias, cuidados periodontales e higiene dental.

3. Después de finalizar la radioterapia se recomienda una buena higiene dental, fluoraciones periódicas y frecuentes controles estomatológicos.
4. Los enjuagues orales diarios con clorhexidina pueden disminuir la incidencia de caries y de gingivitis. Asimismo, el eliminar de la dieta los productos de bollería y dulces es una buena medida para disminuir la incidencia de caries a largo plazo.

5. Debido a la falta de saliva, la mucosa oral está más seca y propensa a irritarse por la comida. Por ello, es aconsejable que aquellos pacientes que no toleren alimentos ácidos (tomate, naranja, limón, vinagre, etc) los eliminen de la dieta y beban agua con frecuencia durante las comidas. Algunos pacientes refieren una mejoría de los síntomas cuando acompañan las comidas con bebidas más viscosas, como la leche o los zumos no ácidos. Platos de comida cocinados con salsas no ácidas mejoran las molestias causadas por la deglución del bolo alimentario.

6. En los intervalos entre las comidas se pueden adoptar también toda una serie de medidas. Beber agua con frecuencia para mantener las mucosas hidratadas, facilita también la higiene oral. Algunos pacientes refieren beneficio con el uso de caramelos sin azúcar o de goma de mascar.

7. Sustitutivos de la saliva. Los sustitutivos de la saliva están comercializados en diferentes presentaciones: sprays, geles y líquidos. Su efecto desgraciadamente es poco duradero (aproximadamente unos 15 minutos), lo que asociado al hecho de que no se contempla su adquisición en régimen de Seguridad Social hace que sean pocos los pacientes que los utilizan de modo continuo y suelen ser reemplazados por sorbos frecuentes de agua. Estos sustitutivos de la saliva pueden contribuir a aliviar la sequedad nocturna que en algunos pacientes puede ser muy importante $e^{9-11,20-23}$.

\section{Tratamiento de la xerostomía con estimulantes salivares}

Se han utilizado diferentes estimulantes salivares desde hace unos años para reducir el grado de xerostomía. Entre ellos el más conocido y utilizado ha sido la pilocarpina $\left(\right.$ Salagen $\left.^{\circledR}\right)$. Es un agonista colinérgico que produce un rápido incremento del flujo salivar, siendo la secreción salivar de características 
similares a las de la saliva normal. Su efecto se inicia a los 30 minutos de su ingesta y se mantiene entre una y tres horas. En los pacientes irradiados por cáncer de cabeza y cuello su eficacia se ve limitada por el hecho de que la pilocarpina requiere de la presencia de tejido glándular sano. A partir de los $60 \mathrm{~Gy}$, la capacidad de la pilocarpina para estimular la producción de flujo salivar es muy limitada; en esta situación, los beneficios que los pacientes refieren se atribuyen a un leve incremento en la producción de saliva por parte de las glándulas salivares menores, ricas en componente mucoso y con función lubricativa de la cavidad oral. Los estudios aleatorizados que muestran beneficio por el uso de la pilocarpina se basan en escalas y pruebas subjetivas obtenidas del paciente ${ }^{9,10,12,21,23-32}$. La pilocarpina ofrecerá mejores resultados en aquellos pacientes con menor xerostomía o menor porcentaje de glándulas salivares irradiado; así pues, aquellos pacientes en los que mediante técnicas conformacionales y de intensidad modulada se consiga preservar un volumen adecuado de glándulas salivares, serán los casos idóneos para obtener un beneficio relevante con mejoría en su flujo salivar. Esto reviste especial relevancia teniendo en cuenta que esta tecnología será implementada en los Servicios de Oncología Radioterápica en los próximos años. Además, hay que destacar que la pilocarpina se ha mostrado útil en un estudio al disminuir la incidencia de candidiasis durante la irradiación ${ }^{33}$. Algunos autores han publicado su utilidad cuando se administra desde el inicio de la radioterapia, atribuyéndosele un efecto protector de las glándulas salivales, aunque no se dispone de resultados a largo plazo. Se han descrito efectos indeseables asociados con la administración de dosis iguales o superiores a $5 \mathrm{mg} / 3$ veces al día. Los más frecuentes son sudoración, lagrimeo y rinitis, aunque es posible la aparición de otros como hipotensión, y su aparición puede llevar a la interrupción del tratamiento. Para evitarlo, la administración puede efectuarse incrementando progresivamente el número de comprimidos hasta un total de 3 al día. Se aconseja una duración mínima de 8-12 semanas para poder evaluar su eficacia. Éste fármaco está contraindicado en pacientes con glaucoma, iritis aguda, asma no controlado, tratamientos con antagonistas betaadrenérgicos, anticolinérgicos o aquéllos con acción parasimpaticomimética $^{10,11,21,27 .}$

\section{Tratamiento preventivo de la xerostomía}

\subsection{Radioprotectores}

El más recientemente incorporado en la clínica por la efectividad que se le ha atribuido ha sido la amifostina $\left(\right.$ Ethiol $\left.^{\circledR}\right)$. Es un radioprotector cuyo metabolito WR-1065 se acumula selectivamente en las glándulas salivares y es el que ejerce el efecto preventivo del daño radioinducido. Diferentes autores han llevado a cabo estudios cuyos resultados han mostrado beneficios en reducir la xerostomía aguda y crónica ${ }^{34-43}$. Después de que se publicase como recomendación de la ASCO en 1999, muchas instituciones la incorporaron para prevenir la xerostomía en los tratamientos con radioterapia del cáncer de cabeza y cuello ${ }^{44,45}$.

En general, se acepta una reducción del grado G2 de xerostomía en un $40 \%$ de los casos, sin grandes aumentos en el flujo salivar. La evidencia atribuida a la utilidad de la amifostina en la prevención de la xerostomía, basada en los diferentes estudios, ha sido de nivel ${ }^{36-38,40-42,46}$. Sin embargo, el perfil de los efectos secundarios, su alto coste y la necesidad de su administración endovenosa, antes de cada sesión de radioterapia, ha limitado su uso extenso en la práctica clínica y levantado polémica en cuanto a su utilidad. Jens Overgaard, en una publicación reciente, refiere como resultado de su análisis que los estudios efectuados hasta la actualidad no son suficientes como para justificar el nivel de evidencia I que se le ha atribuido ${ }^{47}$. Otros autores establecen como argumento que la evidencia no es suficiente, como para justificar el uso sistemático de la amifostina en la radioterapia del cáncer de cabeza y cuello, y que requiere un mayor número de estudios controlados bien diseñados. A pesar de ello, hasta la actualidad, la amifostina es el único fármaco que ha mostrado un nivel aceptable de evidencia en la reducción del grado de xerostomía. Más recientemente, se ha extendido el uso de la amifostina por vía subcutánea, que si bien estudios en ratas sugieren igual eficacia que la vía endovenosa, y algunos autores han referido beneficio en disminuir el grado de xerostomía, los efectos secundarios de este medicamento pueden ser importantes y, al igual que la administración endovenosa, requiere también de estudios bien diseñados para establecer su efectividad ${ }^{40,41,48-53}$. Es de destacar que ha sido referido el beneficio de su administración sub- 
cutánea a mitad de dosis en la radioterapia hiperfraccionada, administrándola antes de cada fracción de tratamiento y con pocos efectos indeseables ${ }^{40}$.

\subsection{Técnicas de radioterapia conformacional $y$ de intensidad modulada (IMRT)}

Con la aparición de las técnicas conformacionales y de intensidad modulada, diferentes autores han publicado sus resultados en la prevención efectiva de la xerostomía. La mejor medida para evitar la xerostomía es no irradiar las glándulas salivares. En este sentido se han efectuado diferentes estudios que, además de mostrar un beneficio evitando su irradiación, muestran por primera vez datos al respecto de la dosis de radioterapia, el volumen irradiado de glándulas salivares y la xerostomía.

Maes y cols. realizaron un estudio en 39 pacientes, en los que mediante técnicas conformacionales se prevenía la irradiación de la glándula parótida contralateral (el 87\% de los pacientes recibieron una dosis igual o menor a 26 Gy en la glándula parótida contralateral). Estos autores refieren la prevención de la xerostomía en el $78 \%$ de los pacientes, utilizando como medios de evaluación las escalas analógicas visuales y LENT-SOMA así como un estudio gammagráfico; concluyen que la dosis en la parótida contralateral tiene que ser igual o inferior a $20 \mathrm{~Gy}$ para obtener una preservación gammagráfica igual o superior al $70 \%{ }^{54}$.

Chao y cols, en 3 estudios publicados entre 2001 y 2002 en los que un reducido número de pacientes fue tratado con técnicas conformacionales e IMRT, ofrecen los siguientes datos: 1) el flujo salivar se reduce un $4 \%$ por cada Gy que reciben las glándulas parótidas, 2) la xerostomía grado 2 de la RTOG es del $22 \%$ y la grado 3 del $0 \%$, en comparación a su serie retrospectiva en la que la xerostomía grado 2 fue del $87 \%, 3$ ) el volumen parotídeo que recibe 30 Gy es inferior utilizando técnicas de intensidad modulada del haz en comparación con la planificación $3 \mathrm{D}^{55-57}$.

Lee y cols. refieren conseguir en 41 pacientes con cáncer de nasofaringe, en los que se administró 5060 Gy con técnicas de intensidad modulada y la sobreimpresión con braquiterapia de alta tasa de dosis, una dosis media de 34 Gy en el 50\% de las parótidas. La xerostomía a los 2 años en estos pacientes según la escala de la RTOG fue: $2 \%$ G2, $32 \%$ G1, $66 \% \mathrm{G} 0^{58}$.
Parliament y cols, en un estudio de 23 pacientes tratados mediante IMRT, muestran que la dosis media y máxima que reciben las parótidas está relacionada con el flujo salivar ${ }^{59}$.

Amosson y cols analizan la xerostomía en 30 pacientes mediante cuestionarios; estos autores concluyen que los parámetros dosimétricos en las glándulas parótidas están relacionados significativamente con el confort general, la ingesta y las alteraciones en el gusto ${ }^{60}$.

Otros estudios posteriores han mostrado también beneficios en la reducción de la xerostomía utilizando técnicas de IMRT, y la mayoría de los autores concluyen que la dosis en una de las parótidas debe ser inferior a 26-30 Gy para evitar la xerostomía ${ }^{61,62}$.

Es de destacar la reflexión de que estos pacientes en los que se ha conseguido preservar las glándulas salivares serán los más beneficiados con la administración de pilocarpina. La amifostina en la actualidad, teniendo en cuenta su coste, necesidad de infraestructura en su administración, sus efectos secundarios y que su eficacia se ha cuestionado por algunos autores, requiere en estos pacientes de estudios amplios bien diseñados que tengan en cuenta la relación coste/beneficio.

\section{Conclusiones y recomendaciones del GETCOCACU para la prevención y la terapéutica de la xerostomía radioinducida}

Las recomendaciones del GETCOCACU en el tratamiento de la xerostomía y sus repercusiones contemplan 4 aspectos: medidas de carácter general, la utilización de estimulantes salivares, incorporación de fármacos radioprotectores $\mathrm{y}$, por último, de técnicas de radioterapia destinadas a evitar la irradiación de las glándulas salivares.

\section{Medidas de carácter general}

1. Mantener una higiene y cuidados dentales específicos antes y después de la irradiación mediante controles estomatológicos/odontológicos. Evitar los alimentos cariógenos, los irritantes orales y los que disminuyan el $\mathrm{pH}$ oral.

2. Hidratación oral frecuente con agua o sustitutivos salivares. 


\section{Estimulantes salivares}

La pilocarpina es el más conocido y al que se le ha atribuido una mayor eficacia. Actualmente está comercializada en España y la dosis recomendada es de $5 \mathrm{mg} / 8$ horas. Su utilidad reside en producir incrementos en la producción de saliva de las glándulas salivares menores, cuando las glándulas salivares mayores han sido destruidas por dosis altas de radiación. El futuro de este medicamento se sitúa sobre todo en aquellos pacientes en los que se pueda llevar a cabo técnicas de IMRT para preservar la irradiación de las glándulas salivares y, probablemente, en disminuir la incidencia de candidiasis durante la irradiación.

\section{Radioprotectores}

Destaca la amifostina, cuyo coste y vía de administración dificulta su utilización extensiva a todos los pacientes con cáncer de cabeza y cuello. La administración subcutánea de este fármaco requiere de estudios fase III para establecer su indicación. En la actualidad son necesarios estudios bien diseñados en los aspectos de eficacia y toxicidad, que tengan en cuenta los histogramas dosis recibida en las glándulas salivares y su volumen o porcentaje irradiado, y de la relación coste/beneficio.

\section{Técnicas conformacionales o de IMRT}

Estos tratamientos son los únicos que van a evitar o reducir la xerostomía. En los próximos años esta tecnología se verá implementada en los Servicios de Oncología Radioterápica de España y muchos de los pacientes con cáncer de cabeza y cuello se podrán beneficiar de ello. Probablemente la pilocarpina aportará a estos pacientes una mejoría sintomática importante al haberse preservado la glandula salivar y la amifostina tendrá su indicación en aquellos pacientes en los que las técnicas conformacionales o de IMRT no permitan la preservación glandular.

\section{Bibliografía}

1. Hazuka M, Martel MK, Marsh L, Lichter AS, Wolf GT. Preservation of parotid function after external beam irradiation in head and neck cancer patients: a feasibility study using 3-Dimensional treatment planning. Int J Radiat Oncol Biol Phys 1993; 27: 731-7.

2. Eisbruch A, Marsh LH, Martel MK, Ship JA, Haken RT, $\mathrm{Pu}$ AT, Fraas BA, Lichter AS. Comprehensive irradiation of head and neck cancer using conformal multisegmental fields: assesment of target coverage and non involved tissue sparing. Int J Radiat Oncol Biol Phys 1998; 41(3): 55968.

3. Fraas BA, Keesler ML, McShan DL, Marsh LH, Watson B, Dusseau WJ, Eisbruch A, Sandler HM, Lichter AS. Optimization and clinical use of multisegment intensity-modulated radiation therapy for high dose conformal therapy. Semin Radiat Oncol 1999; 9(1): 60-77.

4. Grant W, Woo SY. Clinical and financial issues for intensity-modulated radiation therapy delivery. Semin Radiat Oncol 1999; 9(1): 99-107.

5. Nowak PJCM, Wijers O, Lagerwaard FJ, Levendag PC. A three-dimensional CT - based target definition for elective irradiation of the neck. Int J Radiat Oncol Biol Phys 1999; 45(1):33-9.

6. Nishikoa T, Shirato H, Arimoto T, Kaneko M, Kitahara T, Oomori K, Yasuda M, Fukuda S, Inuyama Y, Miyasaka K. Reduction of radiation-induced xerostomia in nasopharyngeal carcinoma using CT simulation with laser patient marking and three-field irradiation technique. Int J Radiat Oncol Biol Phys 1997; 38(4): 705-12.

7. Eisbruch A, Ship JA, Martel MK, Haken RKT, Marsh LH, Wolf GT, Esclamado RM, Bradford CR, Terrell JE, Gebarski SS, Lichter AS. Parotid gland sparing in patients undergoing bilateral head and neck irradiation: techniques and early results. Int J Radiat Oncol Biol Phys 1996; 36(2): 469-80.

8. Fu KK, Pajak TF, Troti A \& cols. Radiation Therapy Oncology group (RTOG) phase III randomized study to compare hyperfractionation and two variants of accelerated fractionation to standart fractionation therapy for head and neck squamous carcinomas: first report of RTOG 9003. Int J Radiat Oncol Biol Phys 2000; 48:7-16.

9. Holmes S. Xerostomia: aetiology and management in cancer patients. Support Care Cancer, 1998; 6:348-55.

10. López P, Bermejo A. Xerostomía en el paciente geriátrico. En: Odontoestomatología Geriátrica. Coordinación Editorial. Madrid 1996; p: 211-28.

11. Guchelaar HJ, Vermes A, Meerwaldt JH. Radiation-induced xerostomia: pathophysiology, clinical course and supportive treatment. Support Care Cancer. 1997; 5: 281-8.

12. Ramirez V, Silverman S, Mayer P, Tyler M, Quivey J. Candidal colonization and oral candidiasis in patients undergoing oral and pharyngeal radiation therapy. Oral Surg Oral Med Oral Pathol Oral Radiol Endod 1997; 84: 149-53.

13. Epstein JB, Chin EA, Jacobson JJ, Rishiraj B, Le N. The 
relationships among fluoride, cariogenic oral flora and salivary flow rate during radiation therapy. Oral Surg Oral Med Oral Pathol Oral Radiol Endod 1998; 86: 286-292.

14. De Vicente JC, Cobo J, Villa MA. Xerostomía post-radioterapia en pacientes con cáncer oral. Modificaciones de los componentes inorgánicos e inmunoglobulinas de la saliva. Avances en Odontoestomatología 1991; 7: 503-17.

15. Davies AN, Singer J. A comparison of artificial saliva and pilocarpìne in radiation-induced xerostomia. J Laryngol Otol 1994; 108: 663-5.

16. Jensen SB, Pedersen AM, Reibel J, Nauntofte B. Xerostomía and hypofunction of the salivary glands in cancer therapy. Support Care Cancer, 2003; 11(4): 207-25.

17. Sanz M, Santos S. Xerostomía o síndrome de boca seca. Periodoncia y Osteointegración 2004; 14(3): 173-82.

18. Anttila SS, Knuuttila ML, Sakki TK. Depresive symptoms as an underlying factor of the sensation of dry mouth. Psichosom Med 1998; 60: 215-18.

19. Hancock P, Epstein JB, Saddler GR. Oral and dental management related to radiation therapy for head and neck cancer. J Can Dent Assoc 2003; 69(9): 585-90.

20. Senahayake F, Piggott K, Hamilton-Miller JMT. A pilot study of salix SST (Saliva-stimulating Lozenges) in postirradiation xerostomia. Curr Med Res Opin 1998; 14(3):155-9.

21. Davies AN. A comparison of artifial saliva and pilocarpine in the management of xerostomia in patients with advanced cancer. Palliat Med 1998; 12: 105-11.

22. Sreebny LM, Zhu WX, Schwartz SS, Meek AG. The preparation of an autologous saliva for the use with patients undergoing therapeutic radiation for head and neck. J Oral Maxillofac Surg 1995; 53:131-9.

23. Joensuu H. Treatment for postirradiation xerostomia. N Engl J Med 1994; 13: 141-2.

24. Horiot JC, Lipinski F, Schraub S, Maylin C, Bensadoun RJ, Gerard JP, Bolla M, Coscas Y, Baillet F, Coche-Dequéant B, Urbajtel M, Montbarbon X, Bourdin S, Wibault P, Pene F, Alfonsi M, Calais G, Desprez P, Favre A, Lapeyre M, Vinke J, Labart M, Savary L. Can pilocarpine hydrochloride relieve xerostomia regardeless of the destruction of major salivary glands? A prospective cooperative study. Radiother Oncol. 1996; 40: S41.

25. Waserman LR, Faulds D. Oral pilocarpine: A review of its pharmacological properties and clinical potential in xerostomia. Drugs 1995; 49(1): 143-55.

26. LeVeque FG, Montgomery M, Potter D, Zimmer MB, Rieke JW, Steiger BW, Gallagher SC, Muscoplat CC. A Multicenter, randomized, double-blind, placebo-controlled, dose-titration study of Oral pilocarpine for treatment of radiation induced xerostomia in head and neck cancer patients. $\mathrm{J}$ Clin Oncol 1993; 11: 1124-31.

27. Jacobs CD, Van Der Pas M. A multicenter maintenance study of oral pilocarpine tablets for radiation induced xerostomia. Oncology $1996 ; 10(3): 16-20$.

28. Cooper RA, Cowan RA, Owens SE, Jeans SP,Roberts JK, Hillel PG, Slevin NJ, Allan E, Gupta N, Coollins CD. Does salivary gland scintigraphy predict response to pilocarpine in patients with post-radiotherapy xerostomia? Eur $\mathrm{J}$ Nucl Med 1999; 26(3): 220-5.

29. Johnson JT, Ferreti GA, Nethery WJ, Valdez IH, Fox FC, David DS, Muscoplat CC, Gallagher SC. Oral pilocarpine for post-irradiation xerostomia in patients with head and neck cancer. N Engl J Med 1993; 329: 390-5.

30. Zimmerman RP, Mark RJ, Tran LM, Juillard GF. Concomitant pilocarpine during head and neck irradiation is associated with decreased postreatmetn xerostomia. Int J Radiat Oncol Biol Phys 1997; 37(3): 571-5.

31. Rieke JW, Hafermann MD, Johnson JT, Leveque FG, Iwamoto R, Steiger BW, Muscoplat C, Gallagher SC. Oral pilocarpine for radiation-induced xerostomia: integrated efficacy and safety results from two prospective randomized clinical trials. Int J Radiat Oncol Biol Phys 1995; 31(3): 661-9.

32. Bloom M, Kopp S, Lundeberg T. Prognostic value of the pilocarpine test to identify patients who may obtain longterm relief from xerostomia by acupuntcture. Arch Otolaryngol Head and Neck Surg 1999; 125: 561-6.

33. Ferre J Viñals H, Rovirosa A, Puig J, Francisco RM, Biete A. Predictive model of the increment of oral C. Albicans in patients subjected to radiotherapy in function to the salivary flowand the use of pilocarpine. J Oral Pathol Med 2004;33(8): 474-5.

34. Brizel DM, Wasserman TH, Henke. Phase III randomized trial of amifostine as a radioprotector in head and neck cancer. J Clin Oncol 1996;14: 2101-12.

35. Buentzel J, Glatzel M, Weinaug R, Schuth J, Kuettner K, Froehlich D. Amifostine in combined radio-and chemomodalities for head and neck cancer. Eur J Cancer 1998; 35(4):363-4.

36. Sauer R, Wannenmacher M, Wasserman T, Henke M, Monier A, Eschwege F, Russell L, Brizel D. Randomized phase III trial on radiation $+/$. amifostine in patients with head and neck cancer. Proceedings of ASCO 1999; Abstract 1516.

37. Shönekäs KG, Wagner W, Prott FJ. Amifostine-A radioprotector in locally advanced head and neck cancer. Eur J Cancer. 35(4): 170.

38. Antonadou D, Bolanos N, Komi P, Kardamakis E, Puglisi M, Antupas G, Karageorgis N, Throuvalas. Effect of amifostine on toxicities associated with induction chemotherapy and radiotherapy for head and neck and lung cancer. Eur J Cancer 35(4): 377.

39. Wasserman T. Radioprotective effects of amifostine. Semin Oncol 1999; 26(2:suppl.7): 89-94.

40. Martinez J, Villar A, Fuentes C, Espiñeira M, Borque C, Hernández R. Feasibility study on twice-a-day fractionated subcutaneous amifostine. Int J Radiat Oncol Biol Phys 2002; 54(2): 312.

41. Bourhis J, Wibault B, Luboinski F, Eschewege F. A randomized phase II study of very accelerated radiotherapy with and without amifostine in advanced head and neck carcinoma (HNSCC). Int J Radiat Oncol Biol Phys 2000; 46(5): 1105-8. 
42. Büntzel J, Kuttner K, Fröhlich D, Glatzel M. Selective cytoprotection with amiphostine in concurrent radiochemotherapy for head and neck cancer. Ann Oncol 1998; 9: 5059.

43. Wagner W, Radmar d A, Schönekaes KG. A new administrtion schedule for amifostine as a radioprotector in cancer therapy. Anticancer Res 1999; 19: 2281. 2284.

44. Hensley ML, Schuchter LM, Lindley. American Society of Clinical Oncology. Clinical practice guidelines for the use of chemotherapy and radiotherapy protectans. J Clin Oncol 1999; (17): 3333-55.

45. Schuchter LM, Hensley ML, Meropol NJ, Winer EP. 2002 update of recommendations for the use of chemotherapy and radiotherapy protectans: clinical practice guidelines of the American Society of Clinical Oncology. J Clin Oncol 2002; 20: 2895-903.

46. Macdonald S. Preliminary results of a pilot study using WR-2721 before fractionated irradiation of the head and neck to reduce salivary gland disfunction. Int J Radiat Oncol Biol Phys 1994; 29(4):747-54.

47. Brizel DM, Overgaard J. Does amifostine have a role in chemoradiation treatment? Lancet 2003; 4(6): 378-81.

48. Shaw L. A phase I study comparing the relative bioavailability of intravenous (IV) and subcutaneous (SC) administration of amifostine (Ethiol). Proceedings of ASCO. 1997; vol.16:250 .

49. Koukourakis M, Fragiadaki C, Stavroulaki A, Ratalis R. Subcutaneous amifostin during fractionated radiotherapy: A randomiced phase II study in pelvic tumors. Eur J Cancer 1999; 35 (suppl.4): 98.

50. Ravo V, Guida C, Silvestro G, Russo F, Elmo M, Pecori B, Frezz P. Subcutaneous administration of amifostine in patients treated with radiotherapy. A preliminary report. Eur J Cancer 1999; 35(suppl.4):102.

51. Anne P, Curran W, Machtay M and cols. A Phase II trial of subcutaneous amifostine and radiation therapy in patients in patients with head and neck cancer. Int J Radiat Oncol Biol Phys 2001; 51(2):150.

52. Cassat DR, Fazenbaker CA, Kifle G, Bachy C. Subcutaneous administration of amifostine is equivalent to intravenous administation in a rat mucositis model. Int $\mathrm{J}$ Radiat Oncol Biol Phys 2003; 3(1): 794-802.

53. Rovirosa A, Ferré J, Verger E, Francisco RM, Fernández J,
Navalpotro B y cols. Amifostina subcutánea en el tratamiento de la xerostomía radioinducida. Revista de Oncología 2003; 5 (Suppl. 4): 128

54. Maes A, Weltens C, Flamen P, Lambin P, Bogaerts R, Liu $\mathrm{X}$, and cols. Preservation of parotid function with uncomplicated conformal radiotherapy. Radiother Oncol 2002; 63: 203-11.

55. Chao C. Protection of salivary function by intensity-modulated radiation therapy in patients with head and neck cancer. Semin Radiat Oncol 2002: 12(1): 20-5.

56. Chao C, Majhail N, Huang C, Simpson JR, Perez CA, Haughey B, and cols. Intensity modulated radiation therapy reduces late salivary toxicity without compromissing tumor control in patients with oropharyngeal carcinoma: a comparison with conventional techniques. Radiother Oncol 2001; 61: 275-80.

57. Chao C, Deasy JO, Markman J, Haynie J Perez CA, Purdy JA. A prospective study of salivary function sparing in patients with head and neck cancers receiving intensity-modulated or three-dimensional radiation therapy: initial results. Int J Radiat Oncol Biol Phys 2001; 49(4): 907-16.

58. Lee N, Xia P, Quivey JM, Sultanem K, Poon I, Akazawa C \& cols. Intensity -modulated radiotherapy in the treatment of nasopharyngeal carcinoma: an update of the UCSF experience. Int J Radiat Oncol Biol Phys 2002; 53(1): 12-22.

59. Parliament MB, Scrimger RA, Anderson SG \& cols. Preservation of oral health -related quality of life and salivary flow rates after inverse-planned intensity modulated radiotherapy (IMRT) for head and neck cancer. Int J Radiat Oncol Biol Phys 2004; 58(3): 663-73.

60. Amosson CM, Teh BS, Van TJ. Dosimetric predictors of xerostomía for head and neck cancer patients treated with the samrt (simultaneous modulated accelerated radiation therapy) boost technique. Int J Radiat Oncol Biol Phys 2003; 56(1): 136-44.

61. Chao C, Ozyigit G, Blanco I \& cols. Intensity-modulated radiation theapy for oropharingeal carcinoma: impact of tumor volume. Int J Radiat Oncol Biol Phys 2003; 59(1): 43-50.

62. Münter MW, Karger CP, Hoffner SG \& cols. Evaluation of salivary gland function after treatment of head and neck tumors with intensity-modulated radiotherapy by quantitative pertechnectate scintigraphy. Int J Radiat Oncol Biol Phys 2004; 58(1): 175-84.
Correspondencia:

Dra. A. Rovirosa

Secretaria del GETCOCACU

Servicio de Oncología Radioterápica

Hospital Clínic i Universitari

C/ Villarroel, 170

E-08036 Barcelona

E-mail: rovirosa@clinic.ub.es 